\title{
DETC2003/DTM-48684
}

\section{FUNCTIONAL, BEHAVIORAL AND STRUCTURAL FEATURES}

\author{
David C. Brown, \\ Al in Design Group \\ Computer Science Department \\ WPI, Worcester, MA 01609, USA.
}

\begin{abstract}
In this paper we examine the definition of the term "feature", and "functional feature" in particular. The goal of the paper is to shed some light on the reason for the profusion of types of features that have been discussed in the literature, to revisit the general definition of the term, and to attempt a definition that uses concepts from Artificial Intelligence. By separating structure, behavior and function, and by defining function, alternative interpretations of "functional feature" are obtained.
\end{abstract}

\section{INTRODUCTION}

In the Engineering community there have been many published definitions of the term "feature" (e.g., see [Salomons 1995] [Shah \& Mantyla 1995] ). This has produced ambiguity and has allowed the introduction of many different types of features.

A confusing proliferation of types of features have been identified, including:

- Form Features: related to the geometric form.

- Precision Features: concerning deviations from nominal dimensions.

- Technological Features: related to performance and operation.

- Material Features: material composition, treatment, conditions.

- Assembly Features: concerning the assembly process.

- Pattern Features: patterns of similar entities.

- Connection Features: geometric constraints.

- Property Features: properties not explicitly related to geometry.

- Application Features: related to process planning requirements.
- Manufacturing features: related to particular processes, such as machining.

However, faced with such variety, and in order to provide a generic definition, writers have gradually been moving towards very general definitions that are in fact only minor variations of "anything about the thing being designed that's of interest” [Dixon 1991].

For example, Vandenbrande \& Requicha [1993] define features as regions of an object that are meaningful for a specific activity or application. Shah [1992], and Shah \& Mantyla [1995], stress that features represent the engineering meaning of the geometry of a part or assembly.

Note that these definitions, along with most definitions of the types mentioned above, relate features back to "regions" or to geometry. That is, they related to physical structure, regardless of the fact that the reason for declaring something a feature varies depending on the type identified (e.g., Assembly, or Connection).

In this paper we attempt to provide a deeper understanding of what it means to be a feature, develop a general definition for features, and discuss what a functional feature is.

\section{FUNCTIONAL FEATURES}

In addition to the types of features mentioned above, another type has been identified-Functional features: concerning function, purpose or behaviors.

Functional features have been increasingly mentioned in the literature. This has happened as CAD has been influenced by Concurrent Engineering, by theories about the design process, and has moved away from being purely geometric. 
McGinnis \& Ullman [1992] write that:

"Functional features include both the purpose of the design object such as support, stability, or strength and the behavior that the design object performs like lifting, gripping, or rotating. The form features embody the physical characteristics of design objects in a design while the functional features explain what purpose the design objects achieve individually and what behaviors they exhibit in the overall design."

This suggests that while a functional feature will have implications for structure/form it is not necessarily about the form.

\section{HOW CAN WE DESCRIBE "ANYTHING”?}

Returning to the generic definition of a feature as "anything of interest", first we discuss how we can describe "anything". Later we consider how can to describe "of interest".

The term "anything" refers to all of, or any realizable portion of, what is being designed. It is represented by a description. Traditionally that description has been geometric. However, a richer and more complete description of an object can be made using a three level Structure-BehaviorFunction (SBF) representation [Sembugamoorthy \& Chandrasekaran 1986].

Hence the term "anything" can refer to all or any realizable portion of an SBF representation.

During designing, the SBF description may be incomplete, or abstract. SBF representations allow for layers of abstraction at the structural and behavioral levels, and they could be extended to allow for functional abstraction as well.

SBF allows for designs to consist of a configuration of nondecomposable elements. Both structures and behaviors can be configurations.

This is all completely consistent with what we see during designing. Design decisions can be very abstract (e.g., "it will be pump-like") or very concrete (e.g., "3.25 inches in diameter"), depending on the design phase. Design decisions can be made about structure:

e.g., shape, components, configuration, material, surface finish, etc.;

about behavior:

e.g., how many states, state-state changes, time taken, flow rates, etc.;

or about function:

e.g., what useful behaviors will be available in what environments under what situations.

There are now many variations of the SBF representation [Umeda \& Tomiyama 1997] [Chandrasekaran 1994]. In the original version (see Figure 1), behaviors are described by state changes, and are linked to descriptions of the structures that are involved. Functions are named, and are described in terms of selected behaviors.

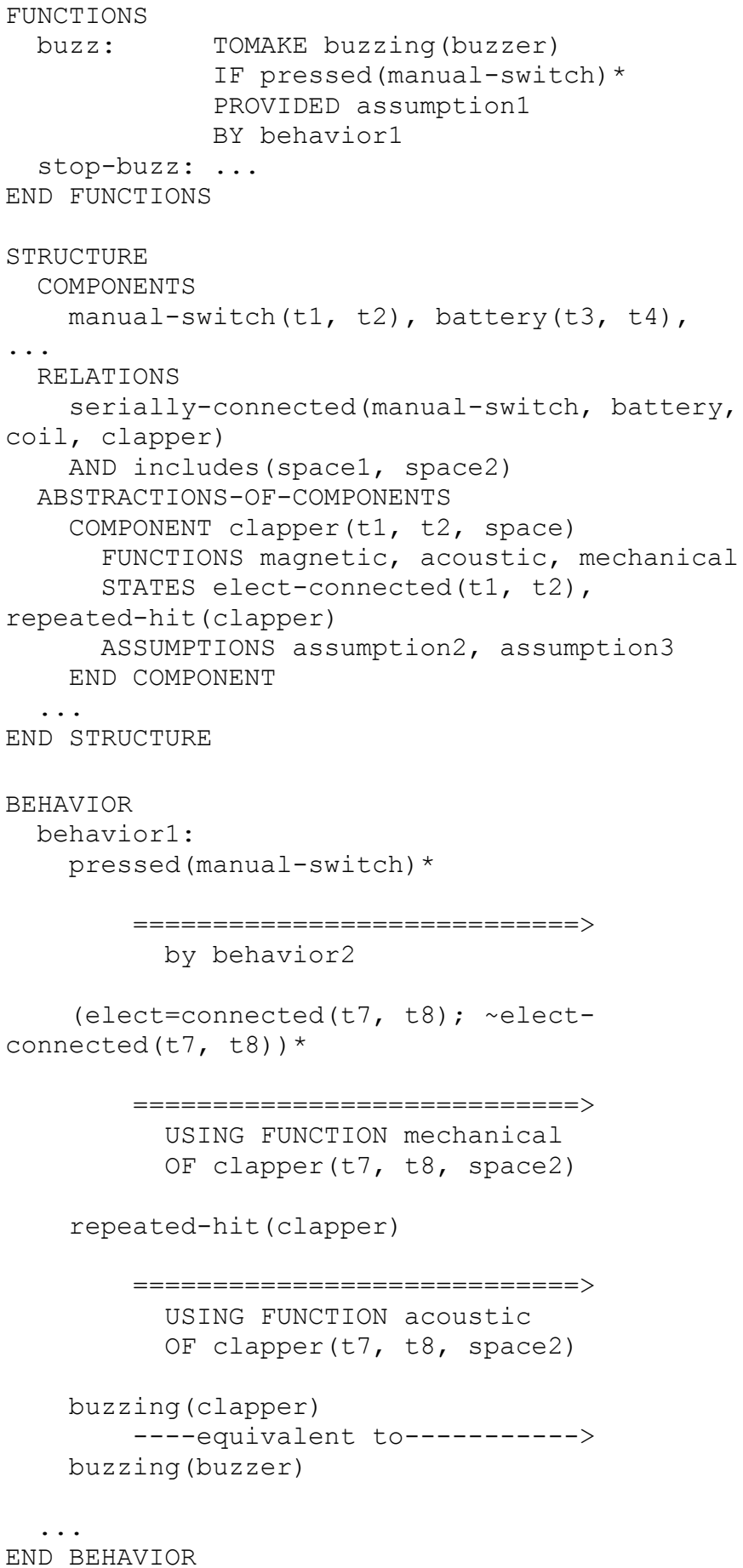

\section{Figure 1: Extracts of SBF representation, for a buzzer, from [Sembugamoorthy \& Chandrasekaran 1986].}

In most functional reasoning research the description of structure is not the main focus of the work, and so the representation normally 'bottoms out' at an abstract description of the types of components and the way they are connected, with no geometric detail. However, nothing would prevent this configuration-oriented level of detail from referring to a geometric level. 
The structural description, $\mathrm{S}$, is considered to include what's needed to describe the physical design, including geometry, spatial relationships, components, and properties. Structural properties, $\mathrm{P}_{\mathrm{S}}$, include material, surface area, surface finish, color, mass, etc.

As far as the author knows, no SBF representation considers the possibility of Behavioral properties, $\mathrm{P}_{\mathrm{B}}$, or Functional properties, $\mathrm{P}_{\mathrm{F}}$. Behavioral properties might include the presence of state-to-state loops, for example, while Functional properties might include whether or not the design has a human user.

\section{FUNCTION}

Function is best thought of in terms of the way that the designed object interacts with an environment when placed in it. Chandrasekaran \& Josephson [1996] define a function of an object in terms of the effect it has on its environment. The environment can use behaviors, structure or structural properties, and can provide 'inputs' or stimuli that trigger the function to enable, prevent or maintain the interaction [Keuneke 1991].

For example, a clock functions when placed in an environment that provides it with power and allows the time display to be visible. Note that in this example a subset of the object's behaviors is being used for a function: the 'ticking' sound is normally not used.

A chair functions, when placed in an environment that places a load on it, by resisting that load. Here the key behavior involves no gross movements and is completely in response to external stimuli.

A knife functions when placed in an environment which applies a force on the knife causing a particular portion of the structure (the 'sharp' edge) to be in contact with another material.

In this last case, the function is provided by a very specific piece of geometry, and by the material properties of the knife that prevent it from bending, breaking or changing its shape. In the clock case, the function is provided by a combination of behaviors and the structure that supports them.

In these examples above we are considering the "intended function" of the designed object, i.e., the design intent. This intended function is concerned with the process we referred to above as "intended use".

Designed objects can be used in other ways (i.e., analogical use) by taking advantage of other substructures, subsets of behaviors, or properties (e.g., using a shoe as a hammer, or using a ticking clock as a baby pacifier).

Refinements to SBF that can be found in the literature include:

- a goal state or control relation that the function is intended to achieve;

- a listing of the properties of the designed object that enable the function;

- a listing of the properties of the environment that enable the function (perhaps including sequences of inputs, or changes in the environment's state);

- the relationships between the properties that enable the function;
- the purpose of the function, described in terms of the environment (i.e., not just the local behavior involved, but its larger impact_-perhaps its design rationale [Lee 1997]).

\section{PROCESSES AND GOALS}

Returning to the clarification of the term "anything of interest", the definitions above from Vandenbrande \& Requicha [1993], Shah [1992] and [Shah \& Mantyla 1995] imply that something is of interest if it is "meaningful for a specific activity" and that this activity must contribute to "engineering meaning".

Hence any activity that engineers should be concerned with while designing can qualify as such an "activity". Shah appears to confirm this when he writes that:

"The engineering significance may involve formalizing the function that the feature serves, or how it can be produced, or what actions must be taken when performing engineering analysis or evaluation, or how the feature "behaves" in various situations."

Rosen et al [1991] define a feature as "a model of the form and intent of some aspect of a design which is of direct interest in a CIM viewpoint". Their "CIM viewpoint" includes Design, Assembly, Manufacturing, Marketing, Analysis, and Service: i.e., any activity that engineers should be concerned with.

Keeping the CIM and Concurrent Engineering approaches in mind, consider all the processes, $\mathrm{P}=\left\{\mathrm{p}_{1}, \mathrm{p}_{2}, \ldots \mathrm{p}_{\mathrm{n}}\right\}$, in which the designed object can be involved.

e.g., intended use, being described, assembling, packing, transporting, diagnosis, simulation, recycling, manufacturing, designing, maintenance, etc.

In order to be "meaningful", and therefore of "interest", we need to consider why someone might be paying attention to one of these processes while designing.

The answer is that the designer $\mathrm{D}$ has goals, $\mathrm{G}_{\mathrm{D}}=\left\{\mathrm{g}_{\mathrm{D} 1}, \mathrm{~g}_{\mathrm{D} 2}\right.$, $\left.\ldots \mathrm{g}_{\mathrm{Dm}}\right\}$, and that she or he is trying to satisfy these goals. A goal, $\mathrm{g}_{\mathrm{Di}}$, could be one of: reduce complexity; reduce resources used; reduce cost; increase reliability; provide ease of use; satisfy requirements; allow access; etc.

The context for each goal, $\mathrm{g}_{\mathrm{Di}}$, is a process, $\mathrm{p}_{\mathrm{j}}$. Each process has a set of compatible goals: i.e., "meaningful for a specific activity". For example:

- one can simplify packing, or simplify describing the design;

- one can reduce the cost of manufacturing, or of designing;

- $\quad$ one can increase reliability during intended use, or during assembly;

- $\quad$ one can allow access for maintenance, or for recycling.

Calculations, simulations, estimates, measurements or heuristic hypotheses may be made to determine if a goal is affected or met. 


\section{DEFINING "INTEREST"}

A designer, $\mathrm{D}$, has at least one point of view (POV). A point of view, $\mathrm{POV}_{\mathrm{Dij}}$, is described by a goal/process pair, $\left(\mathrm{g}_{\mathrm{Di}}, \mathrm{p}_{\mathrm{j}}\right)$.

Interest is relative to a POV. That is, someone has an interest if a design decision or set of decisions might affect the POV's goal in the context of its associated process. The effect might be positive or negative.

For example:

- process choice of a very hard material might increase the cost of the manufacturing;

- choice of a large fan as a component might reduce "ease of use" during the intended use, due to noise and moving air;

- choice of acute angles at edges might reduce "ease of use" during the intended use, due to the possibility of a person being cut;

- choice of acute angles at edges might reduce "ease of use" during packaging, due to the possibility of packaging material being cut;

- choice of friction instead of electromagnetic force as a form of resistance might increase the ability to use the component with electrical devices;

- choice of lack of symmetry might increase handling time; and

- choice of snap fits might decrease assembly time.

Table 1 provides a summary:

Table 1. Examples of POV Goals affected by a Design Decision

\begin{tabular}{llll}
\hline Choice & Goal & Process & Effect \\
\hline material & short time & Manufact'g & -ve \\
component & high ease of use & intended use & -ve \\
geometry & high ease of use & intended use & -ve \\
geometry & high ease of use & packaging & -ve \\
phenomena & high ease of use & intended use & $+\mathrm{ve}$ \\
geometry & short time & handling & $-\mathrm{ve}$ \\
fastener & short time & assembly & $+\mathrm{ve}$ \\
\hline
\end{tabular}

What is of interest to a person depends on the design stage and may be determined by their job/role, by their experiences, or by the information obtained by computer simulation, mental simulation, or by physical modeling of the design.

In most designing, the designer needs to adopt a variety of points of view in order to consider life-cycle issues. Their interest changes as they scan through the stages of the life cycle and, as a consequence, different processes become relevant. Consequently, the relevant features and feature types vary over time.

Note that this reasoning extends to design teams, as in that case different members of the team will have different points of view.

\section{SO, WHAT'S A FEATURE?}

The generic "anything of interest" definition above can be modified now that we know more about both "anything" and "interest". A portion of an SBF description represents "anything", while "interest" concerns a POV.

\section{Definition:}

A feature is described by any portion of an SBF description of the (partially) designed object that may affect the goal of a particular POV.

Note that this clearly means that a feature is dependent on at least one process. Rosen et al [1991] argue, similarly, that a feature contains "relevancy", where "relevancy identifies which CIM viewpoint a feature is defined in".

This definition is consistent with the important notion that a feature is a "view" of the design and that features cannot be listed or "limited a priori" [Shah 1995].

Given this definition, a feature can be described by the S, B or F portions of the description. It corresponds to something in the object being designed, but it isn't always directly about structure.

Hence, for example, a feature can be:

- a structural property: color, size, finish, mass, reflectance, etc.;

- a portion of the surface;

- any portion of the components of the designed object;

- a configuration;

- a set of relationships;

- a behavior, or behaviors; or

- a function, or sub-function.

It may even be a property of a behavior, or of a function. In addition, as SBF is a layered representation, features can be at any level of detail.

\section{GETTING THE RIGHT DESCRIPTION}

A person's POV (e.g., a goal of low duration with respect to manufacturing) determines the portion of the SBF description that is relevant to focus on in order to determine whether a goal has been affected by a design decision or set of decisions.

In addition, a person's POV determines the SBF description built, as alternative ontologies are possible for describing structure or behavior depending on the POV (See Figure 2).

For example, sometimes behavior is better characterized as flow, rather than a sequence of discrete state changes. Another good example is the detection and representation of symmetry in a structural description in response to the goal of keeping handling time low during an assembly process.

Thus the focus of interest changes the SBF description to be considered.

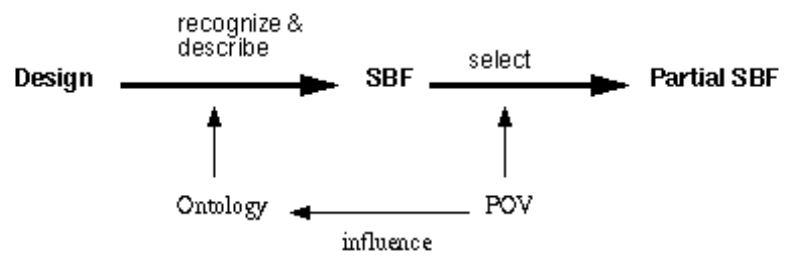

Figure 2. The POV affects the SBF used. 
Note that we consider the SBF description of the design to be distributed, with some of it externally represented (electronically or on paper) and some in the designer's head.

\section{IT'S NOT JUST FOR GEOMETRY ANYMORE}

While many features that fit the definition above refer to 'a region of an object' this issue needs to be discussed. Let us consider some cases:

- Color can be a feature, as it may be of interest because it enables the designed object to function during intended use. Color is an element of the Structural properties, $\mathrm{P}_{\mathrm{S}}$.

- Mass can be a feature, as it may be of interest because it can affect goals in a number of processes, including handling, packaging, and intended use. Mass is an element of the Structural properties, $\mathrm{P}_{\mathrm{S}}$.

- A clock's ticking can be a feature, as it may be of interest because it enables the easy diagnosis of a broken clock mechanism or a lack of power. The "ticking" is a behavior, but not one that is part of the intended function.

- A clock's hands moving can be a feature, as it may be of interest because it enables the designed object to function during intended use. The "moving" is a behavior, and is part of the intended function.

None of these cases refer to a region of the object, and the features aren't geometric. Of course, as function depends on behavior and/or structure, and behavior also depends on structure, everything can be traced back to structure, or to structural properties.

Even then, it's clear that reference back to a "region" isn't always possible. Color for example occupies a region, but is not itself a region or a "shape" [Shah \& Mantyla 1995] - it's just a different 'type'. In the case of "mass", the only possible structure is the whole designed object.

Note that it's clear that, during early stages of the design process, decisions can be made that will cause some impact on the goal of a POV, and can be seen as doing so (i.e., a feature can be detected).

However, at that time only an abstract description of the structure is possible. To insist that features always refer to a region of the object cannot be correct if we take this literally, as early in the design process (especially in non-routine situations) it is possible for no "form" to exist, and for no description of structure to have been committed to.

Shah [1991] recognizes this situation and defines an "abstract feature" as:

"Entities that cannot be evaluated or physically realized until all variables have been specified or derived from the model."

This should not be confused with Shah's 1995 definition of abstract features as higher level classes in a feature taxonomy, even though in some cases the two may be equivalent.

Note that the definition we have developed above has the advantage that it allows abstract features to be of types other than structural.

\section{WHAT'S A FUNCTIONAL FEATURE?}

Up until this point we have considered "function" to refer to the "intended function" of the object being designed. Given the definition of a feature given above we have a choice of what a functional feature might be. It could be a feature that is of type function (i.e., referring to the $F$ portion of the SBF description). It might also be a feature of any type that impacts intended use: e.g., a behavior that prevents the design from functioning. However, this seems less satisfactory.

In an interesting way, every feature is functional. By definition a feature is of interest because it has an effect on a process, and is detected by its effect on a goal. That process is forming an "environment" for that feature, and is interacting with it. This is consistent with the definition of a function of an object as the effect it has on its environment: i.e., by definition, a feature of the designed object is functioning in the environment of each process where that feature affects a goal.

This is not the "intended function" of the designed object, except in the case that the process is "intended use". However, a good designer who is viewing the object from a number of points of view corresponding to the phases of the life-cycle, will ensure that the designed object functions as intended when involved with all the corresponding processes.

For example, it should be easy to pack, easy to maintain, and easy to disassemble. Paying attention to a variety of dynamically detected and selected features during its design make this possible: i.e., it will function well for those processes.

In fact, with respect to functional features, there is a family of types of functions, $F_{p i}$, where $p_{j}$ might be any of the processes already mentioned: i.e., intended use, being described, assembling, packing, transporting, diagnosis, simulation, recycling, manufacturing, designing, maintenance, etc. Clearly, when viewed in this way, all features are functional.

\section{CONCLUSIONS}

In this paper we have examined the definition of the term "feature", and "functional feature" in particular. The goal of the paper is to shed some light on the reason for the profusion of types of features that have been discussed in the literature, to revisit the general definition of the term, and to attempt a definition that uses concepts from Artificial Intelligence.

By separating structure, behavior and function, and by defining function, alternative interpretations of "functional feature" were obtained. The distinctions introduced will allow the development of more flexible use of features in knowledge-based design systems and in designer support systems.

We feel that it is inevitable that knowledge and intelligent use of knowledge will continue to become an increasingly useful component of CAD tools. These tools will know what a designed object is for, and how it works. Computerized simulations of behavior will be augmented by a deeper understanding of how the object interacts with its environment, so that whether it provides its intended function can be checked at design time. Features, as deliverers of function, will play a significant role in this process. 


\section{REFERENCES}

B. Chandrasekaran, "Functional Representation and Causal Processes", Advances in Computers, Vol. 38, Academic Press, 1994, pp. 73-143.

B. Chandrasekaran and J. R. Josephson, "Representing Function as Effect: Assigning Functions to Objects in Context and out", Proc. AAAI-96 Workshop on Modelling and Reasoning about Function, Portland, OR, August 1996.

J. R. Dixon, personal communication, 1991.

A.M. Keuneke, "Device Representation: The Significance of Functional Knowledge", IEEE Expert, Vol. 6, No. 2, Apr. 1991, pp. 22-25.

J. Lee, "Design Rationale Systems: Understanding the Issues", IEEE Expert, Vol. 12, No. 3, 1997, pp. 78-85.

B. D. McGinnis \& D. G. Ullman, "The Evolution of Commitments in the Design of a Component", Journal of Mechanical Design, March 1992, Vol. 114, pp. 1-7. $<w w w . e n g r . o r s t . e d u / \sim$ ullman/evolut1.htm $>$

D. W. Rosen, J. R. Dixon \& X. Dong, “A Methodology for Conversions of Feature-Based Representations", Design Theory and Methodology, DE-Vol. 31, ASME, 1991, pp. 4551.
O. W. Salomons, Computer Support in the Design of Mechanical Products, Ph.D. Thesis, Department of Mechanical Engineering, Universiteit Twente, 1995.

$<w w w . p t$.wb.utwente.nl/staff/otto/thesis/>

V. Sembugamoorthy \& B. Chandrasekaran, "Functional Representation of Devices and Compilation of Diagnostic Problem Solving Systems". In: Experience, Memory, and Reasoning, (Eds.) J. L. Kolodner \& C. K. Riesbeck, Lawrence Erlbaum Associates, Publishers, 1986, pp. 47-73.

J. J. Shah, "Conceptual development of form features and feature modellers", Research in Engineering Design, Vol. 2, 1991, 93-108.

J. J. Shah, "Features in Design and Manufacturing", In: Intelligent Design and Manufacturing, A. Kusiak (Ed.), John Wiley \& Sons, 1992, pp. 39-72.

J. J. Shah \& M. Mantyla, Parametric and Feature-Based CAD/CAM: Concepts, Techniques, and Applications. J. Wiley \& Sons, Inc., 1995.

Y. Umeda \& T. Tomiyama, "Functional Reasoning in Design”, IEEE Expert, special issue on AI in Design, (Eds.) Brown \& Birmingham, Vol. 12, No. 2, March/April 1997.

J. H. Vandenbrande \& A. A. G. Requicha, "Spatial reasoning for the automatic recognition of machinable features in solid models", IEEE Pattern Analysis and Machine Intelligence, Vol. 15, No. 12, pp. 1269-1285, December 1993. $<w w w$-lmr.usc.edu/ jan/Papers/FRec1/NewFRecWeb.html> 